\title{
Complex and Unstable Simple Elbow Dislocations: A Review and Quantitative Analysis of Individual Patient Data
}

\author{
Jeroen de $\operatorname{Haan}^{1}$, Niels Schep ${ }^{2}$, Wim Tuinebreijer ${ }^{2}$ and Dennis den Hartog ${ }^{*}, 2$ \\ ${ }^{I}$ Department of Surgery and Traumatology, Westfriesgasthuis, Maelsonstraat 3, 1624 NP Hoorn, The Netherlands \\ ${ }^{2}$ Department of Surgery-Traumatology, Erasmus MC, University Medical Center Rotterdam, 's Gravendijkwal 230, \\ 3015 CE Rotterdam, The Netherlands
}

\begin{abstract}
Objective: The primary objective of this review of the literature with quantitative analysis of individual patient data was to identify the results of available treatments for complex elbow dislocations and unstable simple elbow dislocations. The secondary objective was to compare the results of patients with complex elbow dislocations and unstable elbow joints after repositioning of simple elbow dislocations, which were treated with an external fixator versus without an external fixator.

Search Strategy: Electronic databases MEDLINE, EMBASE, LILACS, and the Cochrane Central Register of Controlled Trials.

Selection Criteria: Studies were eligible for inclusion if they included individual patient data of patients with complex elbow dislocations and unstable simple elbow dislocations.

Data Analysis: The different outcome measures (MEPI, Broberg and Morrey, ASES, DASH, ROM, arthritis grading) are presented with mean and confidence intervals.

Main Results: The outcome measures show an acceptable range of motion with good functional scores of the different questionnaires and a low mean arthritis score. Thus, treatment of complex elbow dislocations with ORIF led to a moderate to good result. Treatment of unstable simple elbow dislocations with repair of the collateral ligaments with or without the combination of an external fixator is also a good option.
\end{abstract}

The physician-rated (MEPI, Broberg and Morrey), patient-rated (DASH) and physician- and patient-rated (ASES) questionnaires showed good intercorrelations.

Arthritis classification by x-ray is only fairly correlated with range of motion.

Elbow dislocations are mainly on the non-dominant side.

Keywords: Elbow joint, dislocation, fractures, joint instability, therapy.

\section{INTRODUCTION}

The elbow joint is the second most commonly dislocated joint in adults. The annual incidence of simple and complex elbow dislocations in children and adults is 6.1 per 100,000 [1]. Elbow dislocations are classified as simple or complex types [2]. The simple dislocation is characterised by the absence of fractures, while the complex dislocation is associated with fractures. Complex dislocations are associated with fractures of the distal humerus, radial head, ulna or coronoid process. Radial head fractures occur in $36 \%$, coronoid process fractures occur in $13 \%$ and olecranon in $4 \%$ of dislocations of the elbow [1].

Rare fractures in adults include those in the supracondylar humerus, capitellum, and trochlea. Fewer than $2 \%$ of elbow fractures affect the distal humerus. Mehdian

*Address correspondence to this author at the Department of SurgeryTraumatology, Erasmus MC, University Medical Center Rotterdam, 's Gravendijkwal 230, office H-960, 3015 CE Rotterdam, The Netherlands; Tel: +31 107032395 ; Fax: +31 107032396 ;

E-mail: d.denhartog@erasmusmc.nl states that capitellar fractures account for $0.5-1 \%$ of elbow injuries, and trochlear fractures are less common [3].

The radial head and coronoid process are considered to be important bony stabilisers of the elbow. Moreover, an avulsed condylar fracture of the distal humerus may lead to instability due to loss of function of the collateral ligaments [3]. The fundamental goal in the management of fracture dislocation of the elbow is the restoration of the osseousarticular restraints. Therefore, the majority of these complex dislocations are treated with open reduction and internal fixation (ORIF) [4].

Operative treatment of complex dislocations is only described in observational studies [2, 4-8]. However, the surgical goal, namely the restoration of anatomy and early mobilisation, is the same as in other fracture treatments. Assessment of the elbow stability is essential following ORIF of complex elbow dislocations. Signs of instability are redislocation, a positive pivot shift test and positive valgus and varus stress testing. At present, the postoperative management of unstable elbows following ORIF consists of a period of plaster immobilisation in most cases. The 
objective of this immobilisation is to prevent redislocation of the elbow joint and to prevent fracture dislocations [8].

Yet, studies performed in patients with a simple elbow dislocation, i.e. without fractures, indicated that plaster immobilisation exceeding two weeks and following reposition may lead to a limited range of motion [9-11]. Functional treatment could possibly prevent a limited range of motion. Functional treatment is defined as early active movement within the limits of pain with or without the use of a sling or with or without the use of a hinged brace [12, 13].

Theoretically, a period of plaster immobilisation after ORIF of a complex elbow dislocation may result in a limited range of motion and a stiff elbow with subsequent major disability. Theoretically, a hinged external elbow fixator may provide enough stability to start early mobilisation after ORIF of complex dislocations with signs of residual instability [7, 14]. Up to now, only small observational studies about this treatment modality have been published. No randomised controlled trials or observational studies comparing hinged external fixation and immobilisation are available. This is due to the fact that a complex elbow dislocation with remaining instability after ORIF is a rare injury.

The primary objective of this review of the literature with quantitative analysis of individual patient data was to identify the results of available treatment for complex elbow dislocations and unstable simple elbow dislocations. The secondary objective was to compare the results of patients with complex elbow dislocations and unstable elbow joints after reposition of simple elbow dislocations, which were treated with an external fixator versus those treated without an external fixator.

\section{MATERIALS AND METHODOLOGY}

We conducted an electronic search including MEDLINE, EMBASE, LILACS and the Cochrane Central Register of Controlled Trials (CENTRAL). We did not limit the search by language or publication date. We used the following search terms in different combinations as MeSH (Medical Subject Heading) terms and as text words: elbow joint, dislocation, treatment outcome, surgery, controlled clinical trial, and comparative study. Manual searches, including reference lists of all included studies, were used to identify trials that the electronic search may have failed to identify.

Two reviewers independently extracted the data for the primary and secondary outcomes and entered the data into data collection forms developed for this purpose. Discrepancies were resolved by discussion. All data were entered into PASW Statistics (version 17.0; SPSS Inc., Chicago, Illinois). The following variables were retrieved from the studies when available: gender, age, length of follow-up, time between injury and operation, direction of dislocation, side of dislocation, dominance, radial head fracture and treatment, coronoid fracture and treatment, olecranon fracture and treatment, capitellum/tochlea fracture and treatment, terrible triad, external fixator treatment, treatment medial and collateral ligament, ulnar nerve complications, arthritis, contracture release, arthroplasty, ipsilateral injury, pintrack infection, flexion, extension, pronation, supination, motion arc, rotation arc, DASH
(Disabilities of the Arm, Shoulder and Hand) score, Broberg and Morrey score, ASES (American Shoulder and Elbow Surgeons Elbow Evaluation instrument) score, and MEPI (Mayo Elbow Performance Index) score. The DASH, ASES, Broberg and Morrey and MEPI scores range from 0 to 100 points. Higher DASH scores indicate worse upper extremity function, and higher Broberg and Morrey, MEPI and ASES scores indicate better elbow function. The MEPI consists of the physician's assessment of pain, ulnohumeral motion, stability and the ability to perform five functional tasks. The Broberg and Morrey rating system is based on the physician's assessment of motion, strength, stability and pain. The MEPI and Broberg and Morrey scores are ranked categorically according to the following ranks: poor (MEPI and Broberg and Morrey $<60$ points), fair (MEPI 60-74, Broberg and Morrey 60-79), good (MEPI 75-89, Broberg and Morrey 80-94) and excellent (MEPI $>89$, Broberg and Morrey >94). The ASES Elbow Evaluation Instrument combines the patient's assessment of pain, the ability to perform functional tasks and satisfaction with the physician's evaluation of flexion arc, forearm rotation, strength, stability and physical findings. The DASH questionnaire is completed by the patient and evaluates difficulty with performing specific tasks, as well as symptoms, social function, work function, sleep and confidence in relation to the upper limb [15]. Arthritis was classified according to three grades: grade 1 mild, grade 2 moderate and grade 3 severe [16].

In the first analysis, the database was considered as one database and not as a database of different studies. Descriptive statistics were calculated for the complex elbow dislocations and unstable simple elbow dislocations separately. Pearson product-moment correlations were calculated between the continuous outcome measures, and Spearman rho correlations were calculated between the categorical rankings. For the agreement between the MEPI categories and Broberg and Morrey categories, quadratic weighted Kappa correlation coefficients were calculated with StatXact 5 (Cytel Software Corporation). Different variables values between patients with or without an external fixator were compared using unpaired t-tests. The relationship between the motion and rotation arc and treatment with an external fixator was estimated using multiple regression, allowing for the time from injury to operation and length of follow-up. The results are presented as regression coefficients and $95 \%$ confidence intervals (CI). This analysis was stratified by study by including a dummy covariate for each study.

\section{RESULTS}

No randomised trials or comparative studies were retrieved from the literature. Only studies with individual data of the patients were included. The systematic review included 10 observational studies encompassing 170 patients with complex elbow dislocations $(n=134,78.8 \%)$ or unstable simple elbow dislocations $(n=36,21.2 \%)$. Five studies included only complex elbow dislocations [17-21], two studies only simple elbow dislocations [22, 23] and three studies included complex elbow dislocations as well as unstable simple dislocations $[14,24,25]$. The two studies with only unstable simple elbow dislocations published individual data from the same patients $[22,23]$, therefore the 
study with the largest sample size was included [23]. The results of the patients with complex elbow dislocations and the results of the unstable simple elbow dislocations will be presented separately.

\section{Complex Elbow Dislocations}

From 85 of the 134 patients where the gender was retrieved from the articles, 48 were male $(56.5 \%)$ and 37 were female $(43.5 \%)$. The mean age in 105 patients was 41.2 years $(\mathrm{SD}=16.7)$. In 38 patients the side of trauma was noted: in 21 patients the left elbow (55.3\%) was affected, and in 17 cases it was the right elbow (44.7\%). In these 38 cases, the hand dominance was as follows: two patients were $(5.3 \%)$ left-handed and $36(94.7 \%)$ were right-handed. Fifty seven patients $(83.8 \%)$ had a posterior dislocation, 11 patients an anterior dislocation (16.2\%) and in the other 66 patients the dislocation type was not recorded. In eight patients $(6 \%)$ an ipsilateral injury was recorded. Seventy-nine patients $(59.0 \%)$ had coronoid fractures, $112(83.6 \%)$ had radial head fractures, $27(20.1 \%)$ had olecranon fractures and $4(3 \%)$ had capitellum or trochlea fractures. Terrible triad (posterior dislocation with coronoid and radial head fracture) was in seen in 67 patients $(50 \%)$. The mean time in days between the injury and the operation in 34 patients was 13.5 days $(\mathrm{SD}=14.3$; $\min 1, \max 53)$. The mean length of follow-up in months in 103 patients was 87.4 months $(\mathrm{SD}=94.8$; min 10 , $\max 408)$. The operative treatment of the radial head fracture was as follows: ORIF $(n=27,20.1 \%)$, prosthesis $(n=46$, $41.1 \%)$, allograft $(n=1,0.9 \%)$, excision $(n=24,21.4 \%)$ and no operative treatment $(n=14,12.5 \%)$. Eight of the 24 patients with radial head excision were treated wit an external fixator. The treatment of the coronoid fractures was as follows: ORIF or suturing $(\mathrm{n}=43,54.4 \%)$, no operative treatment $(\mathrm{n}=30,38.0 \%)$ and unknown $(\mathrm{n}=6,7.6 \%)$. The 30 coronoid fractures, which were not operated, were in $77 \%$ type I fractures. The treatment of the olecranon fractures was as follows: ORIF $(n=24,88.9 \%)$ and unknown $(n=3,11.1 \%)$. The treatment of the capitellum/trochlea fractures was as follows: ORIF $(n=3)$ and unknown $(n=1)$. In 48 cases the lateral collateral was repaired, and in four cases the medial collateral ligament was repaired. Thirty patients (22.4\%) were treated with an external fixator after treatment of a fracture and/or ligament, except for two cases in which the external fixator was the only operative treatment. One patient was treated with radiocapitellar transfixation with a Kirschner wire. Ten patients $(7.5 \%)$ had a contracture release. Two patients had an arthroplasty (1.5\%) after failed primary treatment, but outcome measures of these patients were not recorded. In five cases the ulnar nerve was released, in one case it was transposed primarily and in seven cases it was transposed secondarily. Two patients had a pin track infection as a result of the external fixator treatment.

Table 1 shows the different outcome measures of the treatment of complex elbow dislocations.

The results of the MEPI categories were as follows: excellent $(n=23,34.8 \%)$, good $(n=26,39.4 \%)$, fair $(n=14$, $21.2 \%)$ and poor $(n=3,4.5 \%)$. The results of the Broberg and Morrey categories were as follows: excellent $(\mathrm{n}=32,27.6 \%)$, good $(n=49,42.2 \%)$, fair $(n=27,23.3 \%)$ and poor $(n=8$,
$6.9 \%$ ). The results of the arthritis Broberg and Morrey categories were as follows: no arthritis ( $\mathrm{n}=34,42.5 \%)$, grade $1(\mathrm{n}=33,41.3 \%)$, grade $2(\mathrm{n}=6,7.5 \%)$ and grade $3(\mathrm{n}=7$, $8.8 \%)$.

Table 1. Different Outcome Measures of the Treatment of Complex Elbow Dislocations

\begin{tabular}{|c|c|c|c|c|}
\hline Outcome Measures & N & Mean & SD & $\mathbf{9 5 \% ~ C I ~}$ \\
\hline \hline Flexion in degrees & 83 & 134.7 & 13.1 & $131.9 ; 137.6$ \\
\hline Extension in degrees & 83 & -17.7 & 13.8 & $-20.8 ;-14.7$ \\
\hline Pronation in degrees & 83 & 67.3 & 23.2 & $62.2 ; 72.4$ \\
\hline Supination in degrees & 83 & 63.8 & 26.8 & $57.9 ; 69.6$ \\
\hline DASH score & 44 & 12.8 & 18.8 & $7.1 ; 18.5$ \\
\hline MEPI score & 37 & 85.7 & 14.9 & $80.7 ; 90.6$ \\
\hline Broberg and Morrey score & 67 & 86.5 & 11.2 & $83.8 ; 89.2$ \\
\hline MEPI category & 66 & 1.95 & 0.87 & $1.74 ; 2.17$ \\
\hline Boberg and Morrey category & 116 & 2.09 & 0.88 & $1.93 ; 2.26$ \\
\hline ASES score & 43 & 89.6 & 13.0 & $85.6 ; 93.6$ \\
\hline Arthritis Broberg and Morrey & 80 & 0.83 & .93 & $.62 ; 1.03$ \\
\hline Motion arc in degrees & 83 & 117.0 & 22.2 & $112.1 ; 121.8$ \\
\hline Rotation arc in degrees & 83 & 131.1 & 44.9 & $121.3 ; 140.9$ \\
\hline
\end{tabular}

The MEPI scores showed excellent agreement with the Broberg and Morrey scores ( $\mathrm{r}=0.90, \mathrm{n}=24)$, ASES scores $(\mathrm{r}=0.84, \mathrm{n}=23)$, and DASH scores $(\mathrm{r}=-0.89, \mathrm{n}=24)$, the Broberg and Morrey scores showed good agreement with the ASES scores $(\mathrm{r}=0.91, \mathrm{n}=23)$ and DASH scores $(\mathrm{r}=-0.84$, $\mathrm{n}=24$ ) and the ASES scores correlated well with the DASH scores $(\mathrm{r}=-0.81, \mathrm{n}=43)$. The Broberg and Morrey categories showed substantial agreement with the MEPI categories (weighted Kappa coefficient $=0.75$, CI $0.63 ; 0.86, \mathrm{n}=53$ ). The motion and rotation, in degrees, showed moderate agreement with the MEPI ( $\mathrm{r}=0.45 ; 0.46, \mathrm{n}=38)$ and Broberg and Morrey $(\mathrm{r}=0.59 ; 0.62, \mathrm{n}=78)$ scores and poor agreement with the ASES $(\mathrm{r}=0.12 ; 0.31, \mathrm{n}=23)$ and DASH $(\mathrm{r}=0.09 ;-0.11, \mathrm{n}=24)$ scores. The agreement (Spearman's correlation) between the arthritis classification from Broberg and Morrey and the MEPI was $0.21(\mathrm{n}=24)$, and the Broberg and Morrey score was $-0.34(n=71)$, and the ASES score was $-0.15(n=43)$, and the DASH score was $-0.06(\mathrm{n}=44)$, and the motion arc was $0.29(\mathrm{n}=91)$, and the rotation arc was $-0.30(\mathrm{n}=71)$. The arthrosis classification from Broberg and Morrey showed slight agreement with the Broberg and Morrey categorical rankings (weighted Kappa coefficient $=0.16$, CI 0.04; 0.29, $\mathrm{n}=91)$ as well as with the MEPI categorical rankings (weighted Kappa coefficient $=0.16$, CI 0.00; 0.39, $\mathrm{n}=44$ )

Table 2 shows the results of the unpaired t-tests comparing patients with versus without an external fixator for the treatment of a complex elbow dislocation.

Tables $\mathbf{3}$ and $\mathbf{4}$ show the results of the multiple regression of the outcome measure motion and rotation arc on the use of an external fixator, time from injury to operation and length of follow-up. 
Table 2. Comparison of Different Variables in Patients without an External Fixator and those with an External Fixator in the Treatment of Complex Elbow Dislocations (Unpaired t-Tests)

\begin{tabular}{|c|c|c|c|c|c|c|}
\hline Variables & No Fixator & N & Fixator & N & 95\% CI Difference & P Value \\
\hline \hline Age in years & $40.5(16.7)$ & 88 & $45.1(16.7)$ & 17 & $-13.3 ; 4.2$ & 0.31 \\
\hline Length follow-up (mths) & $99.5(99.0)$ & 86 & $26.3(20.3)$ & 17 & $49.8 ; 96.5$ & 0.00 \\
\hline Time to operation (days) & $5.5(4.6)$ & 21 & $26.5(15.3)$ & 13 & $-30.4 ;-11.6$ & 0.00 \\
\hline Flexion in degrees & $137(10.9)$ & 66 & $125.9(17.1)$ & 17 & $2.0 ; 20.2$ & $-4.7 ; 10.4$ \\
\hline Extension in degrees & $-17.2(13.9)$ & 66 & $-20.0(13.8)$ & 17 & $-0.2 ; 35.5$ & 0.02 \\
\hline Supination in degrees & $67.4(23.9)$ & 66 & $49.7(33.1)$ & 17 & $.56 ; 34.9$ & 0.052 \\
\hline Pronation in degrees & $71.0(18.9)$ & 66 & $53.2(32.4)$ & 17 & 0.04 \\
\hline Motion arc in degrees & $119.8(19.5)$ & 66 & $105.9(28.6)$ & 17 & $2.3 ; 25.7$ & 0.02 \\
\hline Rotation arc in degrees & $138.3(36.5)$ & 66 & $102.9(62.3)$ & 17 & $2.4 ; 68.4$ & 0.04 \\
\hline MEPI score & $88.8(13.6)$ & 24 & $80.0(16.1)$ & 13 & $-1.4 ; 18.9$ & 0.09 \\
\hline Broberg and Morrey category & $2.02(0.87)$ & 102 & $2.64(0.84)$ & 14 & $-1.11 ;-0.14$ & 0.01 \\
\hline
\end{tabular}

\section{Unstable Simple Elbow Dislocations}

Of the 36 patients 25 were male and 11 were female. The mean age in the 36 patients was 42.6 years $(S D=18.5)$. In 33 patients the side of trauma was noted: in 16 patients the left elbow was affected and in 17 cases the right elbow was affected. In 13 cases the dominance was recorded and was as follows: three patients were left-handed and 10 were righthanded. Fifteen patients had a posterior dislocation, and in the other 21 patients the dislocation type was not recorded. No ipsilateral injury was noted. The time between the injury and operation was only recorded in one patient. In 27 cases the lateral collateral and in 17 cases the medial collateral ligament was repaired; twelve of these cases were bilateral operations. Twenty-five patients were treated with an external fixator, with suturing of the lateral collateral ligament in 18 cases and with suturing of the medial collateral ligament in 13 cases. In two patients, the unstable elbow was stabilised with two Kirschner wires. In the patients without external fixator treatment it was not possible to retrieve exact data about the postoperative period of immobilisation. The mean length of follow-up in months for 34 patients was 29.0 months $(\mathrm{SD}=11.7$; $\min 8, \max 60)$.

In one case the ulnar nerve was released secondarily. One patient had a pin track infection as a result of the external fixator treatment. Two patients had a contracture release.

Table 5 shows the different outcome measures of the treatment of unstable simple elbow dislocations.

The results of the MEPI categories were as follows: excellent $(n=15)$, good $(n=5)$ and poor $(n=1)$. The results of the Broberg and Morrey categories were as follows: excellent $(n=3)$, good $(n=7)$ and fair $(n=1)$. The results of the arthrosis Broberg and Morrey categories were as follows: no arthritis $(n=23)$, grade $1(n=7)$ and grade $3(n=7)$.

Table 3. Effects of External Fixator, Time Between Injury and Operation and the Length of Follow-Up on the Motion Arc in Degrees in 33 Patients with Complex Elbow Dislocations (Multivariate Analysis)

\begin{tabular}{|c|c|c|c|c|}
\hline Variable & Coefficient & $\mathbf{9 5 \%}$ CI & P Value & Standardised Coefficient \\
\hline \hline External Fixator $^{1}$ & -54.0 & $-74.3 ;-33.6$ & 0.000 & -1.1 \\
\hline Time from injury to operation (days) & 1.4 & $0.75 ; 2.1$ & 0.000 & 0.83 \\
\hline Length of follow-up (months) & -0.1 & $-0.26 ; 0.01$ & 0.074 & -0.26 \\
\hline
\end{tabular}

${ }^{\mathrm{T}}$ Compared with no external fixator (reference category).

Table 4. Effects of External Fixator, Time Between Injury and Operation and Length of Follow-Up on the Rotation Arc in Degrees in 33 Patients with Complex Elbow Dislocations (Multivariate Analysis)

\begin{tabular}{|c|c|c|c|c|}
\hline Variable & Coefficient & $\mathbf{9 5 \%}$ CI & P Value & Standardised Coefficient \\
\hline \hline External Fixator $^{1}$ & -102.9 & $-146.1 ;-59.8$ & 0.000 & -1.0 \\
\hline Time from injury to operation (days) & 2.7 & $1.3 ; 4.1$ & 0.001 & 0.77 \\
\hline Length of follow-up (months) & -0.3 & $-0.57 ; 0.00$ & 0.05 & -0.30 \\
\hline
\end{tabular}


Table 5. Different Outcome Measures of the Treatment of Unstable Simple Elbow Dislocations

\begin{tabular}{|c|c|c|c|c|}
\hline Outcome measures & N & Mean & SD & $\mathbf{9 5 \% ~ C I ~}$ \\
\hline \hline Flexion in degrees & 34 & 127.6 & 11.0 & $123.8 ; 131.5$ \\
\hline Extension in degrees & 34 & -13.7 & 9.6 & $-13.7 ;-17.0$ \\
\hline Pronation in degrees & 14 & 69.3 & 16.4 & $59.8 ; 78.7$ \\
\hline Supination in degrees & 14 & 68.6 & 21.4 & $56.2 ; 80.9$ \\
\hline Broberg and Morrey score & 11 & 89.4 & 8.5 & $83.6 ; 95.1$ \\
\hline Arthritis Broberg and Morrey & 31 & 0.32 & .65 & $0.08 ; 0.56$ \\
\hline Motion arc in degrees & 34 & 114.0 & 18.4 & $107.6 ; 120.4$ \\
\hline Rotation arc in degrees & 14 & 137.9 & 33.1 & $118.8 ; 157.0$ \\
\hline
\end{tabular}

Table 6 shows the results of the unpaired t-tests comparing patients with versus without an external fixator for the treatment of an unstable simple elbow dislocation.

\section{DISCUSSION}

This review has included data from 10 observational studies with individual patient data of 170 patients with complex and unstable simple elbow dislocations. Because a lack of randomised controlled trials and comparative studies, no treatment effects could be calculated, and only single group summaries were available. The outcome measures (Tables 1 and 5) show an acceptable range of motion with good functional scores of different questionnaires and a low mean arthritis score. Thus, treatment of complex elbow dislocations with ORIF led to a moderate to good result. Treating unstable simple elbow dislocations with repair of the collateral ligaments with or without the combination of an external fixator is also a good option.

Comparing the patients with a complex elbow dislocation with an additional external fixator versus no external fixator (only ORIF) in univariate analysis showed better flexion, better pronation, and better Broberg and Morrey categories in the patient group that did not receive a external fixator. The patients treated with an additional external fixator had a shorter follow-up time, and the time between trauma and operation was longer. The influence of these two variables was that a shorter follow-up time and a longer interval between trauma and operation was correlated with a higher range of motion. A multivariate regression analysis showed that the motion and rotation was worse in the additional external fixator group when adjusted for the time between trauma and operation and follow-up time. But the external fixator group was small, and the difference between the fixator and non-fixator groups is probably not a real difference but is rather the result of bias; the worst cases were treated with an external fixator. All the patients with the long interval between trauma and fixator placement were from one study [25]. In this study the patients the patients were initially managed in other institutions and the external fixator placement must be interpreted as secondary treatment.

It was not possible to compare patients with or without an external fixator in unstable simple elbow dislocations because of the low sample sizes of the two groups. The only remarkable finding in these patients was the lower mean age in the fixator group (36 versus 57 years). The external fixator was probably reserved for younger patients.

Because no randomised trials and comparative studies were available, we only included studies with individual data. Are our results comparable with reviews of case-series? Many reviews are rather about operative management and give algorithms for treatment $[4,5]$. For instance, if after treating the fractures, the elbow is still unstable, the treatment can be completed with an external fixator or with restriction of motion by protection with a cast or brace. Healing of the fractures is given precedence over mobilisation of the elbow, because chronic instability is more difficult to treat than stiffness. A case-series of 36 terrible triads of the elbow showed after a follow-up period of 34 months a flexion-extension arc of $112^{\circ}$, a forearm rotation of $136^{\circ}$ and a mean MEPI score of 88 points [26]. These patients were treated with a standard surgical protocol [27]. These results are comparable with our analysis.

The physician-rated (MEPI, Broberg and Morrey), patient-rated (DASH) and physician- and patient-rated (ASES) questionnaires showed good intercorrelations. The categorical ranking of the MEPI and Broberg and Morrey showed substantial agreement. Turchin et al. also found good correlations between the MEPI and Broberg and Morrey raw scores (0.89), but their Kappa correlation coefficient between the categorical rankings of the MEPI and Broberg and Morrey was lower $(0.43$, CI $0.26 ; 0.60)$ [28]. Because of this low agreement, they advised the use of raw aggregate scores and not the categorical rankings. Also the correlations of the MEPI and Broberg and Morrey scores with the patient completed functional DASH questionnaire were lower at -0.55 and -0.56 respectively. This difference with our results could be explained by their diverse sample of patients with elbow problems, while our sample was restricted to elbow dislocations. Validation of questionnaires depends on the examined clinical condition [29]. Correlations of the motion and rotation arc were moderate

Table 6. Comparison of Different Variables in Patients without an External Fixator and those with an External Fixator in the Treatment of Unstable Simple Elbow Dislocations (Unpaired t-Test)

\begin{tabular}{|c|c|c|c|c|c|c|}
\hline Variables & No Fixator & N & Fixator & N & 95\% CI Difference & P Value \\
\hline \hline Age in years & $56.8(19.8)$ & 11 & $36.3(14.1)$ & 25 & $8.8 ; 32.3$ & 0.001 \\
\hline Length follow-up (months) & $25.3(16.2)$ & 9 & $30.3(9.7)$ & 25 & $-14.3 ; 4.2$ & 0.28 \\
\hline Motion arc in degrees & $117.2(17.3)$ & 9 & $112.8(18.9)$ & 25 & $-10.3 ; 19.1$ & 0.54 \\
\hline Rotation in degrees & $143.3(33.5)$ & 9 & $128.0(33.5)$ & 5 & $-25.4 ; 56.1$ & 0.43 \\
\hline
\end{tabular}


with the physician-rated (MEPI, Broberg and Morrey) questionnaires and slight with the (partially) patient-rated questionnaires (ASES, DASH). Doornberg et al. also saw moderate correlations between MEPI scores $(\mathrm{r}=0.40 ; 0.38)$ and Broberg and Morrey scores $(\mathrm{r}=0.54,0.50)$ with a range of flexion-extension and pronation-supination, respectively, but their correlations with the DASH and ASES were much higher (flexion-extension $\mathrm{r}=-0.42$ with DASH, 0.56 with ASES; pronation-supination $\mathrm{r}=-0.34$ with $\mathrm{DASH}, 0.50$ with ASES) [30]. Doornberg et al. also examined patients with intra-articular fractures of the elbow, and they found pain and flexion-extension arc as significant predictors for the MEPI, Broberg and Morrey, ASES and DASH scores. Pain even had the strongest influence on the outcome measures. The discrepancy with our correlations between motion and DASH and ASES can be caused by the fact that we only had data from 24 DASH questionnaires and range of motion all from one study [19], in contrast with the sample size of 104 in the study of Doornberg et al. [30].

Arthritis classification was fairly correlated with MEPI, Broberg and Morrey, motion and rotation arc and slightly with DASH and ASES. Josefsson et al. concluded after a mean follow-up of 24 years of 52 simple elbow dislocations that radiographic changes were associated with somewhat decreased extension but rarely caused problems or symptoms [31]. Thus, Josefsson's clinical impressions that degenerative joint changes are slightly correlated with the complaints of the patient are confirmed by the correlations from this review. However, we found no studies that quantitatively correlated arthritis scores of the elbow with functional elbow scores.

The incidence of the radial head, coronoid process and olecranon fractures were much higher in this series than in Josefsson's study [1]. This is caused by the high incidence of medial epicondyle fractures in the patients with elbow fractures of Joseffson's sudy.

In our review, $55 \%$ of the complex elbow dislocations were found on the left side and in Josefsson study 58\% [1]. About only $6 \%$ of a studied American population was left handed [32], so elbow dislocations are mainly found on the non-dominant side. The exact cause of the preponderance of the non-dominant side is unknown, but it could be the result of the employment of the dominant arm during a fall or the unconscious protection of the dominant arm during a fall.

The quantitative analysis of the data in this review showed that a favourable prognosis may generally be anticipated after ORIF of a complex elbow dislocation and of an unstable simple dislocation treated by suturing the collateral ligaments and/or placing an external fixator. The exact role of the external fixator in the treatment of complex elbow dislocations could not be determined from our extracted data. For instance, it would be interesting to know if the external hinged fixator could be used after only minimal ORIF without suturing the collateral elbow ligaments. The benefit of this procedure would be that the patient can start exercising immediately after the operation instead of having the elbow joint immobilised in a plaster for several weeks.

\section{REFERENCES}

[1] Josefsson PO, Nilsson BE. Incidence of elbow dislocation. Acta Orthop Scand 1986; 57: 537-38.

[2] Hildebrand KA, Patterson SD, King GJ. Acute elbow dislocations: simple and complex. Orthop Clin North Am 1999; 30: 63-79.

[3] Mehdian H, McKee MD. Fractures of capitellum and trochlea. Orthop Clin North Am 2000; 31: 115-27.

[4] Ring D, Jupiter JB. Fracture-dislocation of the elbow. J Bone Joint Surg Am 1998; 80: 566-80.

[5] Bain GI. A review of complex trauma to the elbow. Aust N Z J Surg 1999; 69: 578-81.

[6] Lee DH. Treatment options for complex elbow fracture dislocations. Injury 2001; 32(Suppl 4): SD41-SD69.

[7] McKee MD, Bowden SH, King GJ, et al. Management of recurrent, complex instability of the elbow with a hinged external fixator. J Bone Joint Surg Br 1998; 80: 1031-6.

[8] Ring D, Jupiter JB, Zilberfarb J. Posterior dislocation of the elbow with fractures of the radial head and coronoid. J Bone Joint Surg Am 2002; 84-A: 547-51.

[9] Mehlhoff TL, Noble PC, Bennett JB, Tullos HS. Simple dislocation of the elbow in the adult. Results after closed treatment. J Bone Joint Surg Am 1988; 70: 244-49.

[10] Protzman RR. Dislocation of the elbow joint. J Bone Joint Surg Am 1978; 60: 539-41.

[11] Schippinger G, Seibert FJ, Steinbock J, Kucharczyk M. Management of simple elbow dislocations. Does the period of immobilization affect the eventual results? Langenbecks Arch Surg 1999; 384: 294-97.

[12] Maripuri SN, Debnath UK, Rao P, Mohanty K. Simple elbow dislocation among adults: a comparative study of two different methods of treatment. Injury 2007; 38: 1254-58.

[13] Rafai M, Largab A, Cohen D, Trafeh M. Pure posterior luxation of the elbow in adults: immobilization or early mobilization. A randomized prospective study of 50 cases. Chir Main 1999; 18: 272-78.

[14] Stavlas P, Gliatis J, Polyzois V, Polyzois D. Unilateral hinged external fixator of the elbow in complex elbow injuries. Injury 2004; 35: 1158-66.

[15] Longo UG, Franceschi F, Loppini M, Maffulli N, Denaro V. Rating systems for evaluation of the elbow. Br Med Bull 2008; 87: 13161.

[16] Broberg MA, Morrey BF. Results of delayed excision of the radial head after fracture. J Bone Joint Surg Am 1986; 68: 669-74.

[17] Broberg MA, Morrey BF. Results of treatment of fracturedislocations of the elbow. Clin Orthop Relat Res 1987; 109-19.

[18] Egol KA, Immerman I, Paksima N, Tejwani N, Koval KJ. Fracturedislocation of the elbow functional outcome following treatment with a standardized protocol. Bull NYU Hosp Jt Dis 2007; 65: 26370 .

[19] Forthman C, Henket M, Ring DC. Elbow dislocation with intraarticular fracture: the results of operative treatment without repair of the medial collateral ligament. J Hand Surg (Am) 2007; 32: 1200-9.

[20] Lindenhovius AL, Brouwer KM, Doornberg JN, Ring DC, Kloen P. Long-term outcome of operatively treated fracture-dislocations of the olecranon. J Orthop Trauma 2008; 22: 325-31.

[21] Nalbantoglu U, Kocaoglu B, Gereli A, Aktas S, Guven O. Open reduction and internal fixation of Mason type III radial head fractures with and without an associated elbow dislocation. J Hand Surg (Am) 2007; 32: 1560-68.

[22] Jeon IH, Kim SY, Kim PT. Primary ligament repair for elbow dislocation. Keio J Med 2008; 57: 99-104.

[23] Micic I, Kim SY, Park IH, Kim PT, Jeon IH. Surgical management of unstable elbow dislocation without intra-articular fracture. Int Orthop 2009; 33: 1141-47.

[24] Duckworth AD, Ring D, Kulijdian A, McKee MD. Unstable elbow dislocations. J Shoulder Elbow Surg 2008; 17: 281-86.

[25] Yu JR, Throckmorton TW, Bauer RM, Watson JT, Weikert DR. Management of acute complex instability of the elbow with hinged external fixation. J Shoulder Elbow Surg 2007; 16: 60-7. 
[26] Pugh DM, Wild LM, Schemitsch EH, King GJ, McKee MD. Standard surgical protocol to treat elbow dislocations with radial head and coronoid fractures. J Bone Joint Surg Am 2004; 86-A: 1122-30.

[27] McKee MD, Pugh DM, Wild LM, Schemitsch EH, King GJ. Standard surgical protocol to treat elbow dislocations with radial head and coronoid fractures. Surgical technique. J Bone Joint Surg Am 2005; 87(Suppl 1): 22-32.

[28] Turchin DC, Beaton DE, Richards RR. Validity of observer-based aggregate scoring systems as descriptors of elbow pain, function, and disability. J Bone Joint Surg Am 1998; 80: 154-62.
[29] Zarins B. Are validated questionnaires valid? J Bone Joint Surg Am 2005; 87: 1671-72.

[30] Doornberg JN, Ring D, Fabian LM, Malhotra L, Zurakowski D, Jupiter JB. Pain dominates measurements of elbow function and health status. J Bone Joint Surg Am 2005; 87: 1725-31.

[31] Josefsson PO, Johnell O, Gentz CF. Long-term sequelae of simple dislocation of the elbow. J Bone Joint Surg Am 1984; 66: 927-30.

[32] Luetters CM, Kelsey JL, Keegan TH, Quesenberry CP, Sidney S. Left-handedness as a risk factor for fractures. Osteoporos Int 2003; 14: $918-22$

(C) de Haan et al.; Licensee Bentham Open.

This is an open access article licensed under the terms of the Creative Commons Attribution Non-Commercial License (http://creativecommons.org/licenses/by$\mathrm{nc} / 3.0 /$ ) which permits unrestricted, non-commercial use, distribution and reproduction in any medium, provided the work is properly cited. 